Bislang existiert keine Evidenz bezüglich eines Therapeutikums, das zur Prophylaxe der sekundären Schädigung (Hypoxie, Ödementwicklung, Inflammation, Exitotoxizität) nach einer Rückenmarksverletzung zum Einsatz kommen könnte. Deshalb stellt Minocylin eine sehr interessante Substanz dar, der protektive Eigenschaften zugeschrieben werden, und die somit potenziell Eingang in die klinische Routine finden könnte. Allerdings konnte in dieser Studie keine signifikante Verbesserung des neurologischen Outcomes im Behandlungsarm festgestellt werden. Ferner - beim Fokus auf die Zervikalläsionen - lässt sich nicht exakt nachvollziehen, welche Patienten dazu beigetragen haben, ein vermeintliches Annähern an das Signifikanzniveau zu erreichen; es kann sein, dass der beschriebene Effekt nicht so stark ausfällt. Jedoch sollte die sich wohl anschließende Phase-III-Studie mit Spannung erwartet werden. Eventuell liefert diese - neben validen und hierfür gepowerten klinischen Endpunktdaten - weitere, klinisch relevante Aufschlüsse zu den bislang ungeklärten Pro- zessen einer sekundären Myelonschädigung nach akuter Rückenmarksverletzung.



Dr. med. Joji Kuramatsu, Erlangen

Universitätsklinik für Neurologie, Erlangen E-Mail: joji.kuramatsu@uk-erlangen.de (im Bild)

PD Dr. med. Hagen Huttner, Erlangen

Universitätsklinik für Neurologie, Erlangen E-Mail: hagen.huttner@uk-erlangen.de

\title{
Magnesium entfaltet keine neuroprotektive Wirkung
}

Fragestellung: Ist Magnesium bei der Subarachnoidalblutung neuroprotektiv wirksam?

Hintergrund: Die Prognose der Subarachnoidalblutung ist weiterhin schlecht. Die Sterblichkeit innerhalb eines Monats beträgt 27 - 44\%; von den Überlebenden sind $20 \%$ auf Dauer behindert. Die wichtigste Komplikation ist die zerebrale Ischämie durch Vasospasmen. Reblutungen sind heute durch die modernen operativen und interventionellen Techniken des Aneurysmaverschlusses deutlich seltener geworden. Die einzig bisher zugelassene wirksame Therapie zur Prävention von Angiospasmen ist Nimodipin. Magnesium hat neuroprotektive Eigenschaften und ist im Tierexperiment auch in der Lage, Vasospasmen bei Subarachnoidalblutungen zu verhindern. Daher sollte in einer großen Phase-III-Studie untersucht werden, ob Magnesium die Prognose nach Subarachnoidalblutung verbessert.

Patienten und Methodik: Es handelt sich um eine große randomisierte, placebokontrollierte Studie, die in Europa und Südamerika durchgeführt wurde. Patienten im Alter von über 18 Jahren mit einer Subarachnoidalblutung wurden entweder mit $64 \mathrm{mmol}$ intravenösem Mag-

Dorhout Mees SM, Algra A, Vandertop WP et al. Magnesium for aneurysmal subarachnoid haemorrhage (MASH-2): a randomised placebo-controlled trial. Lancet 2012; 380: 44-9
Ergebnisse: Insgesamt wurden 1.204 Patienten in die Studie eingeschlossen. 606 Patienten erhielten Magnesium und 597 Patienten bekamen Placebo. Für den primären Endpunkt hatten 158 Patienten einen schlechten Outcome in der Magnesiumgruppe verglichen mit 151 in der Placebogruppe (26,2\% vs. 25,3\%). Dieser Unterschied war statistisch nicht signifikant.

Auch eine Metaanalyse von sieben randomisierten Studien mit insgesamt 2.047 Patienten zeigte keinen Nutzen von Magnesium im Vergleich zu Placebo im Hinblick auf eine Verbesserung der Prognose nach Subarachnoidalblutung.

Schlussfolgerung: Die intravenöse Gabe von Magnesium ist bei der Behandlung der akuten Subarachnoidalblutung nicht wirksam.

\section{- Kommentar von Prof. Hans-Christoph Diener}

\section{Zerplatzte Hoffnung}

Mit der MASH-2-Studie ist leider die Hoffnung auf eine Prophylaxe und Therapie angiospastischer Insulte bei der Subarachnoidalblutung zerplatzt. Magnesium ist zwar im Tierexperiment wirksam und hatte in kleineren Phase-II-Studien einen positiven Trend bei Patienten mit Subarachnoidalblutungen gezeigt. Wie so häufig in den letzten Jahren ergibt sich dann in einer großen Phase-III-Studie, dass der neuroprotektive Ansatz nicht funktioniert. Daher beschränkt sich die Prophylaxe von angiospastischen Insulten weiterhin auf die Gabe von Nimodipin. 